\title{
Modification of a Visual Monitoring platform via the Power Carrier combining with senior multimedia
}

\author{
Chaoxiong $\mathrm{Li}^{1, \mathrm{a}}$, Tingting Wang ${ }^{1, \mathrm{~b}}$, Heming Cheng ${ }^{1, \mathrm{c}}$, Junyi Yin ${ }^{1, \mathrm{~d}}$, Jingli Gao ${ }^{1}$, \\ Tao Qin ${ }^{2}$ and Yinghui Han ${ }^{1, *}$ \\ ${ }^{1}$ North China Electric Power University, Baoding, 071003, China \\ ${ }^{2}$ Tianjin Power Supply Bureau, State Grid Corporation, Tianjin, 300000, China \\ *Corresponding Author: Yinghui Han (yh_han@126.com)

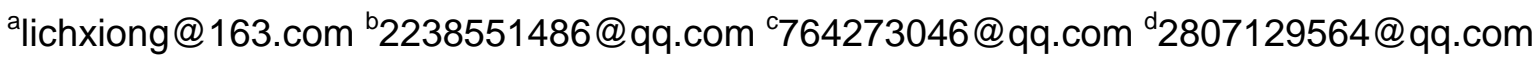

Keywords: Visual monitor; Power Line Communication; OMAP Starter Kit; real-time transmission

\begin{abstract}
To avoid the disadvantages of the traditional video surveillance, such as short distance transmission and one-way flow of analog signals, a modified visual monitoring platform was developed by the power line carrier communication and the senior multimedia technology in this work. The platform made from three parts: the central controller, the operating system and the real-time visual transmission system, respectively. Based on the OMAP5912 chip, a corresponding software program was designed, including the U-BOOT migration, the core system cutting and transplantation, DSP/BIOS system application, the driver design of USB camera and some basic sub-unit and so on. Finally, the host computer browser the video surveillance through the embedded Web BOA server and its characteristics were summarized, the system problems were also proposed.
\end{abstract}

\section{Introduction}

With the rapid development of the embedded multimedia and network technology, the moving high-definition video surveillance, the intelligence and convenience-oriented direction, the data processing required for increasing in the entire video surveillance system. The traditional video surveillance, the video processing and the transmission mode are meeting the new demands and challenges. Under the new requirements, the traditional analog CCTV surveillance system has a lot of disadvantages due to one-way flow and signal transmission distance in the cable segment defects gradually being replaced by digital monitoring system. The currently popular digital network monitoring system was often seen as a good solution to video surveillance in signal processing and transmission distance and speed issues, but it also has a lot of bottlenecks. For example, the "last mile" in the cable distribution network became a wiring terminal drawback during the installation, especially in the re-wiring parts which have already completed works. Although the wireless network solves the network terminal wiring problem, other issues including the uneven distribution of signals, the signal blind spots, and the signal radiation restrict its applications.

At present, power line carrier PLC (Power Line Communication) technology has matured, and now a number of international chip manufacturers have launched $14 \mathrm{Mb} / \mathrm{s}, 85 \mathrm{Mb} / \mathrm{s}$ and $200 \mathrm{Mb} / \mathrm{s}$ rates PLC chip, PLC technology gradually from the previous single remote meter reading to further aspects multimedia applications. Using power line carrier communication technology to access and power line as the transmission medium of video surveillance systems, low cost, no additional wiring and easy installation

According to the unique strengths and deficiencies of traditional video surveillance power line carrier technology and distribution network, the paper proposed based on OMAP5912 dual-core processors and power line carrier's video surveillance system, the dual-core processor used in video surveillance systems while using power line carrier module to transmit video data. Design utilizing dual-core processor ARM side were mobilized and control, DSP end system for processing video data. And the use of power line carrier module for data collected in real-time video transmission. 


\section{The overall design scheme of the system}

The overall scheme of the system diagram was shown in Figure 1. The system mainly includes 3 parts: OSK (OMAP Starter Kit) control system, PLC data transmission module and the host computer monitoring. OSK control system is mainly responsible for the operation of embedded operating system, the video signal acquisition and processing, the data exchange of dual core and the data communication between the power carrier modules, which is an important part of the whole system. Electric power carrier module acts as the OSK system video data collected in the transmission power line. The electric power carrier module can realize real-time transmit the video data collected, will directly affect the overall performance of the whole system.. The host computer monitoring system is mainly responsible for the real-time data transmission for display and storage work of the power carrier module. The design of the whole system has the following a few key points:

(1) drive of USB camera and the video information acquisition;

(2) system dual core communication and the compression of video

(3) The real-time video data transmission of power line carrier communication.

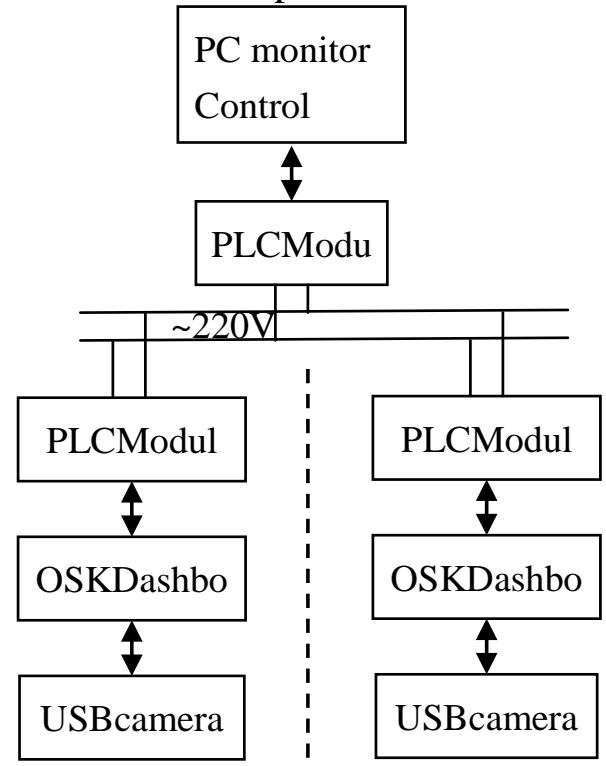

Fig.1 Overall block diagram of a system diagram program.

\section{OSK control panel}

The control board of the monitoring system on power line is not only responsible for the system, task scheduling, acquisition and processing of the video data of video ,but also the need for real-time communication with PLC module. The system adopts Montavista Linux operating system, in order to save the required operating system running the data, such as $U$ Boot, the system parameters, DSP / BIOS, Linux kernel and file system, and the system needs to have external expansion Flash support. Besides, in order to improve the speed of system running, system extended buffer is required. Therefore, the author uses TI company's OSK control as a control panel board system with dual core controller, OMAP5912 as the main control chip, which is a highly integrated software and hardware processor, consisting of a control functions of ARM92 processor and high performance and low power consumption of 1 'Ms320C55xDSP, compared to the single core system - OMAP5912 a significant advantage is able to greatly improve the operation efficiency of the system. The OSK control board not only has the external expansion interface commonly used ,such as serial port, JTA port, USB port and network interface, also includes DDR SDRAM 32 M Flash and $32 \mathrm{M}$. Voltage of various external expansion chip in the system adopt different, such as the main control core OMAP5912 using $1.6 \mathrm{~V}$ low power voltage, USB and serial port voltage of 5 $\mathrm{V}$, Flash and Ethernet port voltage of $3.3 \mathrm{~V}$, so a stable and reliable power supply is the key of the stable operation of the system. The system uses TPS65010 low voltage linear regulator to 
management system of power supply, it not only can meet a variety of output different voltage, and a static current minimum $3.2 \mathrm{uA}$ in the static work, which can meet the demand of low power embedded system and can work stably in a wide range of temperature. The DSP power circuit was shown in Figure 2.

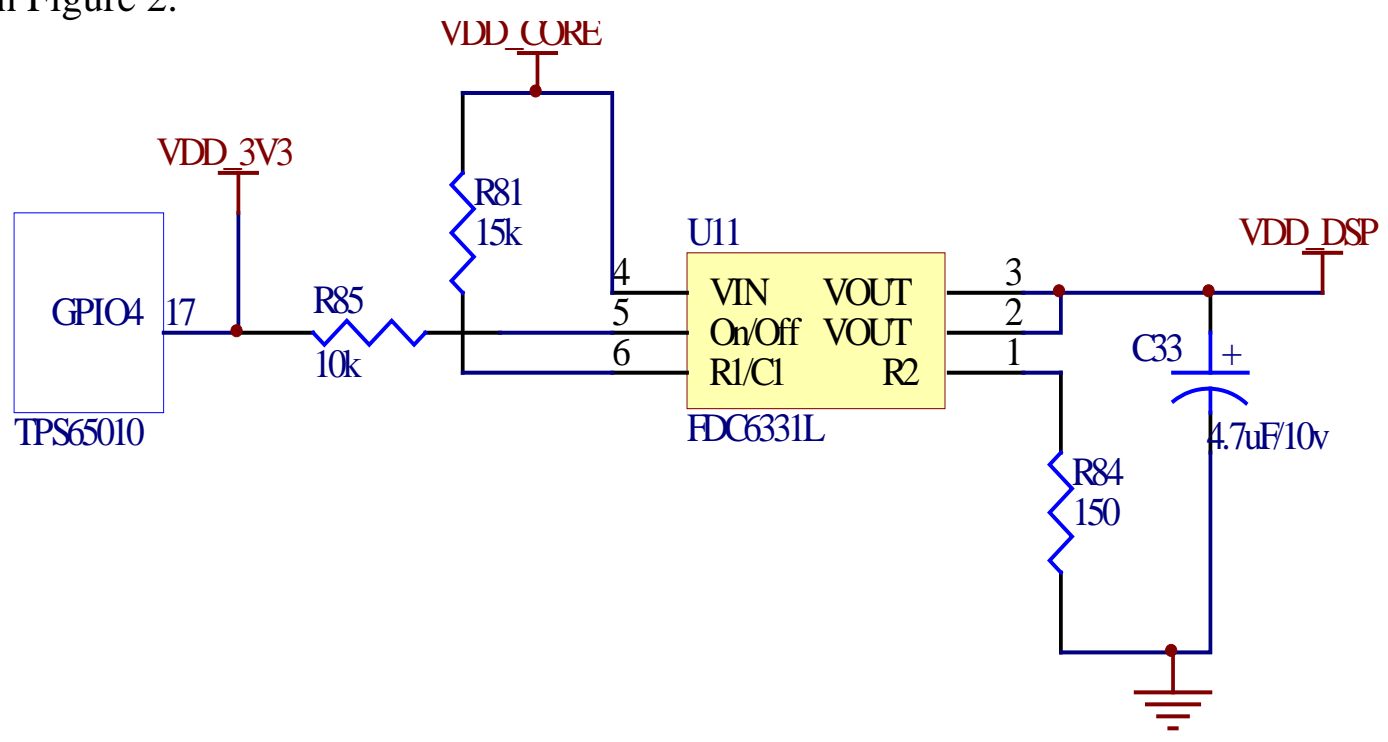

Fig.2 DSP power circuit

\section{Power carrier module}

The system by using power line as the transmission medium is because it has no wiring, network covering a wide range, has the advantages of convenient connection, less investment and quick effect etc.. The system lower position machine of the power carrier module system mainly communicate with the main control board by data and the video data package received into the power line carrier frame for transmission on power line. The power carrier module of upper machine mainly receives the power line carrier frame on the line, and it restores the transmission of video data compression to the host computer monitoring system. In order to ensure the ability of real-time monitoring of video monitoring system, the system to choose the power carrier module of HomePlu91.0 protocol based on NT5500 chip, the module maximum transmission speed of up to $85 \mathrm{Mb} / \mathrm{s}$, the function of $\mathrm{H} 1$ with multi points broadcasting. The theory module far transmission distance is $300 \mathrm{~m}$ and extended to support a maximum of 128 of the road, but the actual test use in the environment because of the problem of interference sources, transmission distance between modules can guarantee the ideal speed within $100 \mathrm{~m}$, when the distance exceeds $100 \mathrm{~m}$, the transmission rate of the module decreases with distance. The module is directly connected through the network interface and the control panel, the basic connection was shown in Figure 3.

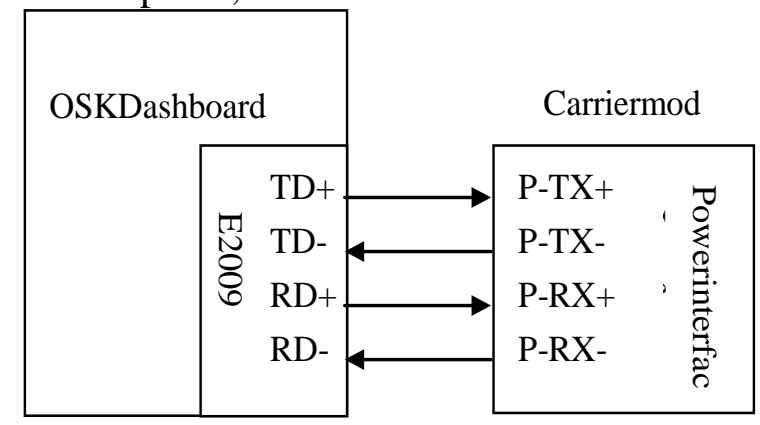

Figure.3 Power line carrier module connection diagram

\section{Software Design}

System software design includes the establishment of a development environment, USB camera-driven development, communications, video data compression and communication between the dual-core module drives. The system will use the widely used open-source Linux operating system for the system to collect video data via USB, network modules use the same power line 
carrier communication module. Dual-core TI's communications system adopted DSP / BIOS Software Development Kit. System main program flow chart shown in Figure 4.

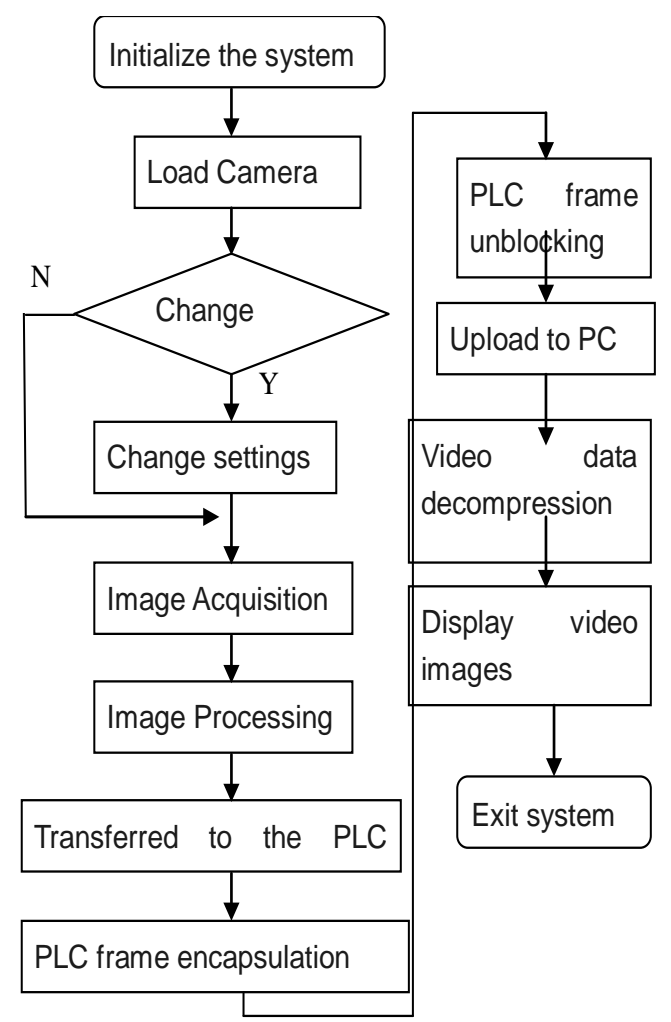

Fig.4 Main program flow diagram

\section{Video image acquisition}

\section{1) USB camera driver}

In order to make the system can support different USB camera, we use an open-source USB camera driver package SPCA5XX. SPCA5XX now on the market which includes the majority of the camera driver. SPCA5XX source package consists of four main components: the equipment module initialization module and unload modules, top software interface module, data transmission module [5]. The entire code is executed from the device module initialization module module_init (usb_spca5xx_init), in order to unload the module module_exit equipment modules (usb_spca5xx_exit) end. SPCA5XX transplant had to OSK5912 control platform for kernel Kconfig, Makefile and other documents to be modified, but we will need the USB Multimedia devices under SPCA5XX compiled into the kernel. In addition, USB camera driver should work, also need to Multimedia devices-> Video For Linux and USB support-> Support for Host-side USB-> USB Host Controller Drivers -> OHCI HCD support compiled into the kernel.

\section{2) Video image acquisition}

Video equipment belonging to the device file in Linux, after the completion of USB camera driver, can be found in Linux device file that corresponds with the camera too: / dev / video0, then we can be like an ordinary file access to read and write operations [6] [7]. Video capture test code:

insmod spca5xx.o // Load camera driver;

mknod /dev/video0 c 810 ; // Set the connection point;

capture_init("/dev/video0",\&myvideo,320,240);// Initialize the camera configuration mmap_init(\&myvideo,*vd);// Storing an image of the current address mapping myvideo.frame_current $=0$;// Initialize the current number of frames save_picture(get_picture(\&myvideo),"frame.nb.jpg");// Save the camera image captured capture_close(\&myvideo);// Turn off the camera

\section{3) Dual-core data processing}


System uses a dual-core communication software specifically for TI's dual-core processor DSP real-time operating system developed by DSP / BIOS II, it can through DSP / BIOS Link of specific API functions to achieve the end of GPP links with ARM [8]. API functions can be achieved through the operation of the DSP initialization signal processing, task switching, data flow information exchange, query management DSP tasks and resource conditions. After configuring the DSP / BIOS Link to GPP compilation compilation follows:

\#source /dsplink/etc/host/scripts/Linux/gppenv // Setting environment variables

\#cd /dsplink/gpp/src // Change the environment path

\# gmake -s [debug | release] // Began compiling

After successful compilation will be created following the path of the kernel module and user library function:

/dsplink/gpp/export/BIN/Linux/OMAP/DEBUG

/dsplink/gpp/export/BIN/Linux/OMAP/RELEASE

Completion of the above steps and can achieve data communication between the dual-core, dual-core between data processing flow chart shown in Figure 5:

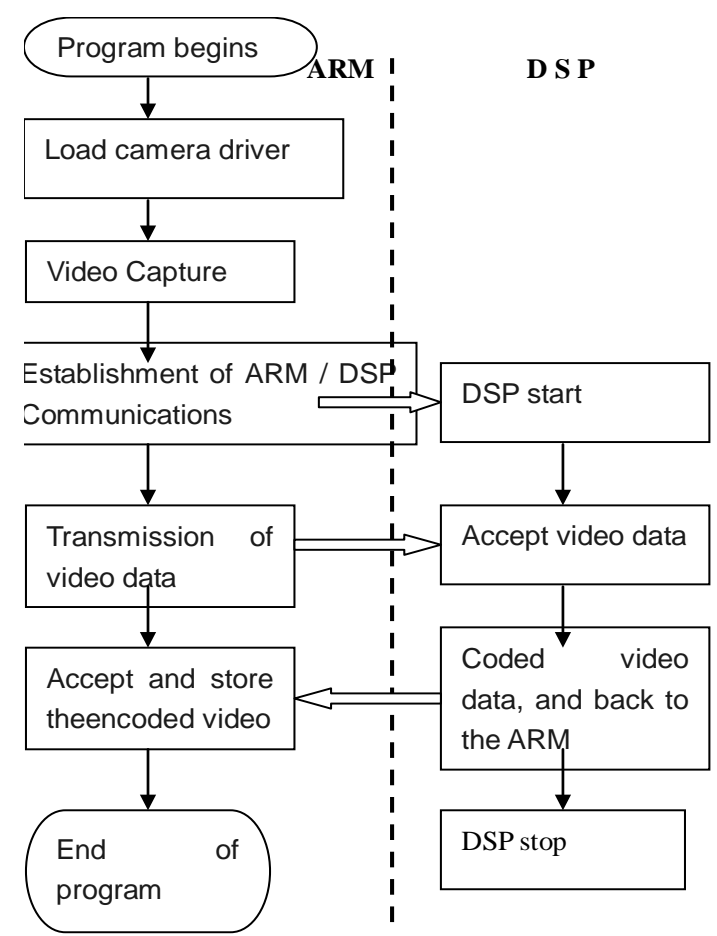

Fig.5 Dual-core communication flow diagram

\section{Communication module}

In order to ensure the timeliness and reliability of video surveillance in the communication module uses a real-time transport protocol RTP / RTCP, the agreement is based on the traditional TCP / IP protocol to support efficient data transmission [9]. Taking into account the performance of other network expansion and maintenance of all aspects of the design will be using C / S (cilent / server client / server) network transport model [10], between the control module and a power transmission module and a power transmission module will establish the appropriate TCP / IP link between the network and the host computer, based on real-time data transmission protocol for data communications and system control. Server SendPacket sends RTP data, function to process the received RTCP SR packet by Poll. Client link with the server through Scoket established after the address and port access by detecting the correct client-server. The client sends a request to the server data, the server receives the request after receiving the data, while the decompressed image data and displayed on the screen. 


\section{Summary}

Implementation of a power line carrier systems for the transmission of video surveillance design, and good will OMAP5912 dual-core technology combined with the power line carrier technology for video surveillance provides a new transmission. Solve the cable network "last mile" problem and the wireless network signal blind spots and other issues. The current chip carrier transmission distance and scalability as well as limitations, particularly impenetrable different transformer electricity network, how to improve the transmission rate and transmission distance power line carrier chip dual-core systems while improving the utilization of the system design will be the next step to be solved problem. The advantages of the extensive coverage of the power grid and convenient way to plug and play video surveillance power line carrier make the system a wide application prospects.

Note: Main context in this work has been published in Journal of WUT (Information \& Management Engineering), 2012, 34(1), 24, in Chinese. In this paper, we modified some parts of the previous system. This paper used mainly in international conference communication.

\section{Acknowledgements}

The research was supported by National Natural Science Foundation of China (No.51308212 and No. 10974053), the National Key Technology R\&D Program (No.2011BAI02B03), the Fundamental Research Funds for the Central Universities (No.13ZD18) and the State Scholarship Fund (No. 201206735009).

\section{References}

[1] G. Wang: Integrated platform based on next-generation internet technology power line communications architecture --PLC, IPv6 and QoS systems. Power system communication, Vol 1.10 (2005),p. 9

[2] C. Peng, C. Yang and Y. Pan.: Principle Open Multimedia Application Platform -OMAP processors and application. Beijing: Electronic Industry Press (2005)

[3] L. Fei,J.li and S.Zhu: OMAP5912 dual-core architecture-based embedded system and its application [J] electrical technology, Vol6 (2006), p.11

[4] S. Chai, S. Hou and X. Cheng: Design and Realization of Broadband Line Communication Modem Based on INT5500. Low Voltage Apparatus. Vol.3 (2007) , p.41

[5] T. Wang,X. Ji: Using a USB camera. The embedded Linux platform Microcomputer Applications, Vol 1 (2000), p.52

[6] Video4Linux Users Manual [EB/OL]. Information on http://www.v4l.com.cn

[7] video4linux USB camera-based image acquisition to achieve. [EB / OL]. http://www.hhcn.org/maindoc/USB_Camera_base_on_Video4Linux.pdf

[8] TI DSP/BIOS Real-time Operating System v6.x User's Guide [EB/OL].Information onhttp://focus.ti.com.cn/cn/dsp/docs/litabsmultiplefilelist.tsp?sectionId=3\&tabId=409\&literatureNu mber=spruex3d\&docCategoryId=6\&familyId $=477$

[9] L. Cao, J. Jiang and M. Qi: RTP protocol design and implementation of embedded network camera [J] Computer Technology and Development, Vol18, (2008),p.214

[10]J. Li, W. Hao: Research and design of Embedded Net2 work Video Monitoring System Based on Linux[J].Computer Science and Software Engineering, Vol 9 (2008),p.1310 